\title{
Fluorescein as a diagnostic marker of bladder ruptures: an experimental study on rabbit model
}

\author{
Özgür Aksoy ${ }^{1}$, Başak Kurt ${ }^{1}$, Celal Şahin Ermutlu ${ }^{1}$, \\ Kürşat Çeçen ${ }^{2}$, Sadık Yayla ${ }^{1}$, Metin Ekinci ${ }^{3}$, \\ İsa Özaydin ${ }^{1}$, Süleyman Erdinç Ünlüer ${ }^{2}$ \\ ${ }^{1}$ Faculty of Veterinary Medicine Department of Surgery, \\ ${ }^{2}$ Faculty of Medicine Department of Urology, \\ ${ }^{3}$ Faculty of Medicine Department of Ophtalmology, \\ Kafkas University, TR-36300, Kars, Turkey \\ sahinermutlu@hotmail.com
}

Received: April 13, 2015 Accepted: May 10, 2016

\begin{abstract}
Introduction: The aim of this study was to investigate fluorescein use in the diagnosis of bladder ruptures in rabbits as an experimental model. Material and Methods: The study was conducted on male New Zealand rabbits divided into a retrograde fluorescein group $(n=8)$ and an intravenous (IV) fluorescein group $(n=8)$. Following general anaesthesia, $10 \mathrm{~mL}$ of $10 \%$ fluorescein dye (sodium fluoresceine powder) was administered via ureterorenoscope to the bladder of the first group, and $0.5 \mathrm{~mL}$ of $10 \%$ fluorescein was administered intravenously to the second group. Then, the bladder was viewed through the cystoscope by urethral aspect. After experimental bladder perforation, groups were comparatively evaluated by paracentesis and laparotomy. Results: Following IV injection of fluorescein dye, the bladder veins were stained green within $10 \mathrm{~s}$ and then fluorescein mixed with urine flowed into bladder lumen. The green fluid flow was observed in the abdominal cavity after the perforation of the bladder in both groups. Conclusion: Fluorescein can be used as a marker in diagnosis of bladder ruptures. If there is no bleeding or intestinal content in the abdominal cavity, although a smoky yellow-green image is observed, bladder rupture can be suspected.
\end{abstract}

Keywords: rabbit, bladder rupture, fluorescein, cystoscopy, catheterisation.

\section{Introduction}

Urinary bladder rupture frequently occurs in animals as a result of trauma or obstruction of bladder outflow in domestic animals. Rupture of the urinary bladder and subsequent uroperitoneum is a common problem in cattle. Diagnosis of urinary bladder rupture can be confirmed readily in dogs and cats by performing retrograde positive contrast cystography or an intravenous contrast study of the urinary system (19). Retrograde positive contrast cystography is rarely rewarding in cattle with urolithiasis because of the urethral obstruction. For this reason, veterinarians depend on certain characteristic electrolyte and biochemical abnormalities to confirm a clinical diagnosis of urinary bladder rupture in cattle.
The release of urine into the peritoneal space leads to dilutional hyponatraemia and hypochloraemia as these ions pass down a concentration gradient from the extracellular space into the peritoneal cavity $(10,19)$. Metabolic acidosis is the usual sequela to urine retention in carnivores but both alkalosis and acidosis have been reported in cattle with ruptured bladders (19).

Uroperitoneum can be suspected on the basis of the history and clinical signs which include depression, progressive abdominal distension, decrease or absence of urination, and hypovolaemia. Demonstration of hyponatraemia and hypochloraemia is supportive of the diagnosis, but the diagnosis is confirmed when comparisons between plasma and peritoneal fluid creatinine concentrations show that ratio to be $2: 1$ or greater in horses (19). 
Fluorescein is a stable, non-toxic, low molecular weight, dibasic acid that diffuses easily into tissues. It is readily buffered to physiologic $\mathrm{pH}$, fluoresces intensely, and is readily measured in biologic substrates by spectrofluorometry (7). Intravenous fluorescein is the most widely used as a contrast agent (17). Fluorescein sodium is used extensively as a diagnostic tool in the field of ophthalmology, and is available in $5 \mathrm{~mL}$ volume at $10 \%$ concentration. Intravenous fluorescein angiography is performed for the management of vitreoretinal diseases by injecting fluorescein sodium dye as a bolus into a peripheral vein $(2,6,14)$.

Fluorescein is a non-toxic agent and it is rapidly absorbed from the peritoneal cavity $(3,7)$. It has been reported that the adverse effects of fluorescein angiography are milder, and are limited to temporary green discoloration of the skin and orange-green discoloration of the urine $(2,7,14)$.

The aim of this study was to investigate whether fluorescein that is administered intravenously and retrograde could be used as a marker without any complications in the diagnosis of bladder ruptures in animals in field conditions.

\section{Material and Methods}

Animals. This experimental model included two groups of adult male New Zealand rabbits, eight rabbits in each group. Preference was given to animals that had full urinary bladders. The first group was named the "retrograde fluorescein group" and the second group was named the "intravenous (IV) fluorescein group".

Anaesthesia protocol. Animals were sedated with $5 \mathrm{mg} / \mathrm{kg}$ of Xylazine $\mathrm{HCl}$ (Rompun ${ }^{\circledR} 2 \%$; Bayer, Germany) and anaesthetised with $35 \mathrm{mg} / \mathrm{kg}$ of Ketamine $\mathrm{HCl}$ (Ketasol ${ }^{\circledR} 10 \%$; İnterhas, Turkey) intramuscularly for $30 \mathrm{~min}$.

Administration of fluorescein. A rigid ureterorenoscope $(6.5 \mathrm{FR} \times 67 \mathrm{~cm}$, Wolf, Germany) and cold light source (Wolf, Germany) was used for the cystoscopy procedure. First, Lidocain $\mathrm{HCl}$ lubricant gel (Cathejell ${ }^{\circledR}$; Taymed, Turkey) was administered to the external urethral orifice.

In the first group, $10 \mathrm{~mL}$ of $10 \%$ fluorescein dye (sodium fluoresceine powder) were administered via ureterorenoscope to the bladder and $0.5 \mathrm{~mL}$ of $10 \%$ fluorescein (Fluorescite ${ }^{\circledR}$; Alcon, Switzerland) was administered intravenously via yellow 24 G IV catheter in the second group. Then, the bladder was viewed through the cystoscope by urethal aspect.

Experimental bladder perforation. The back wall of the bladder was perforated with endoscopic scissors (4 FR, Wolf, Germany) and transition of fluorescein from the bladder to abdominal cavity was confirmed with cystoscopic camera.

Paracentesis. A paracentesis procedure was performed following bladder perforation from caudal to the umbilicus by inserting a green $21 \mathrm{G}$ puncture needle cranially into the abdomen when the animals were in lateral recumbency. Some fluid sample was aspirated from abdominal cavity by paracentesis.

Laparotomy. After the cystoscopy, rabbits were shaved from the xiphoid process of the sternum to the pubis, extending laterally for laparotomy. A midline incision was made through the skin from caudal to the xiphoid down to the pubis. The tissues were dissected routinely and abdominal cavity and organs were examined.

Treatment protocol. The bladder incison was closed with routine suture techniques after the abdominal examination, and abdominal drainage was performed. Adequate hydration and electrolyte balance were maintained and treatment with gentamicin sulfate (Genta 20; I.E. Ulagay, Turkey) was continued for seven days. All animals were hospitalised during the healing period after the surgical procedure.

\section{Results}

Fluorescein reached the bladder veins within $10 \mathrm{~s}$ after intravenous administration in the second group and the veins became green (Fig. 1A). Then fluorescein mixed with urine flowed into the bladder lumen. (Fig. 1B). Fluorescein which was administered intravenously was found to be in arteries after $5 \mathrm{~s}$ and veins after 7-20 s and extravasation was seen after 30-120 s. Urinary excretion of fluorescein started after 5-10 min. This indicated that fluorescein reached the bladder lumen in a short time (Fig. 1C).

After perforation of the bladders in both groups, the green fluid flowed from the perforated bladders into the abdominal cavity (Fig. 1D).

The fluid was aspirated from the abdominal cavity via paracentesis in both groups following cystoscopy-guided bladder incision. Thus, bladder rupture was confirmed. The flow of the green fluid from the bladder into the abdominal cavity was observed during laparotomy. All internal organs were stained yellow-green (Fig. 2).

There was no coloration of the conjunctiva and mucous membranes in the retrograde fluorescein group which was given fluorescein into the bladder directly (Fig. 3A), while in the intravenous fluorescein group, the colour of the conjunctiva, sclera and mucous membranes changed to fluorescent yellow (Fig. 3B). These symptoms were considered to be adverse effects. 


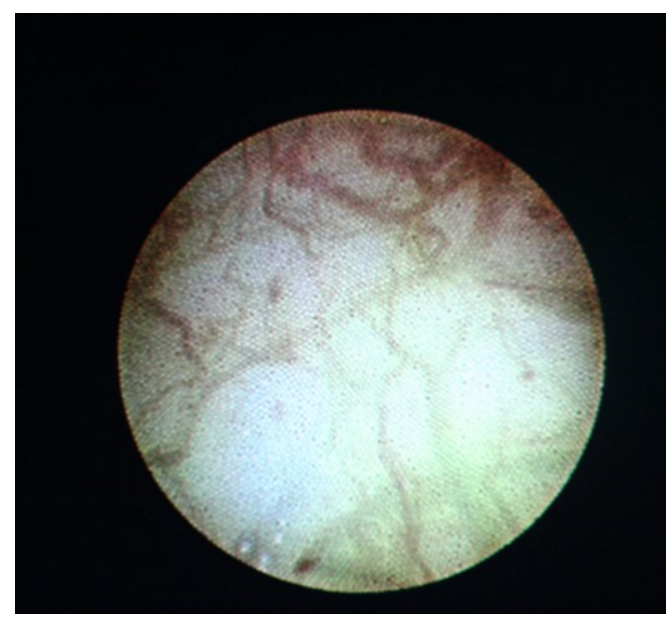

Fig. 1A. The veins of bladder mucosa stained green after administration of fluorescein in the intravenous fluorescein group

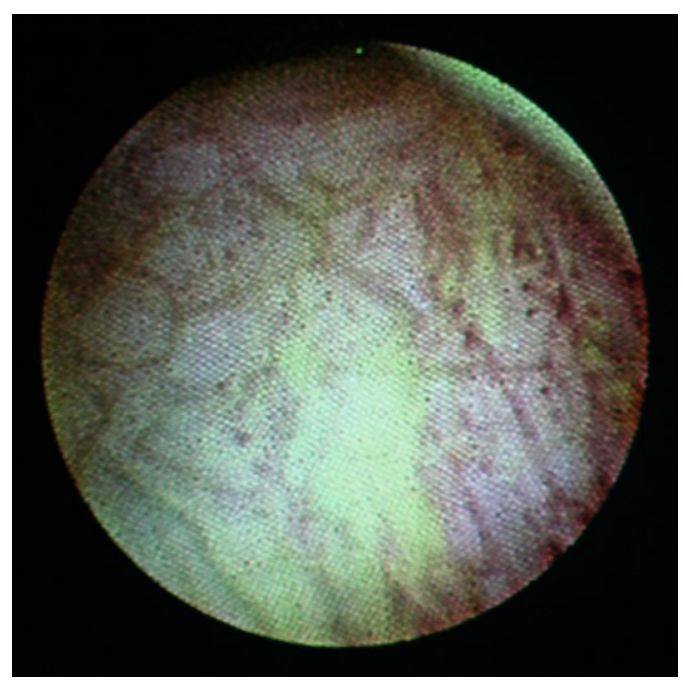

Fig. 1B. Green fluid flow started into the bladder in intravenous fluorescein group

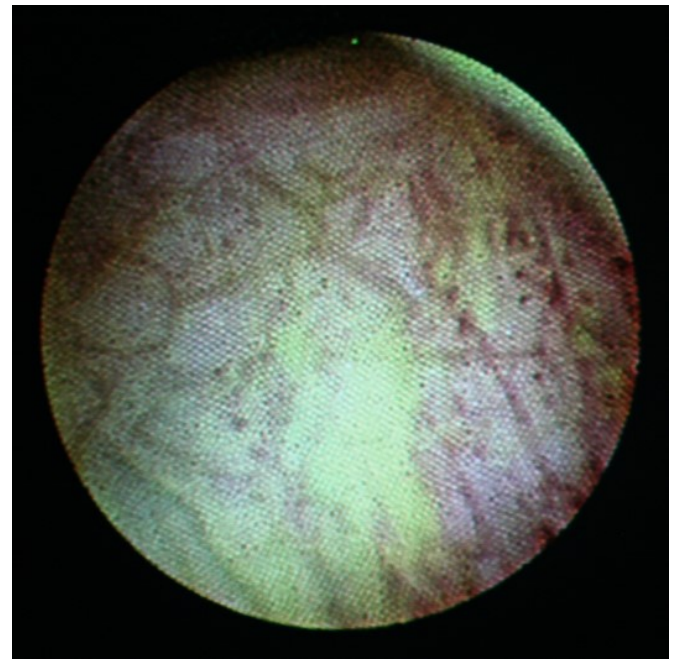

Fig. 1C. Appearance of the filled urinary bladder with fluorescein dye

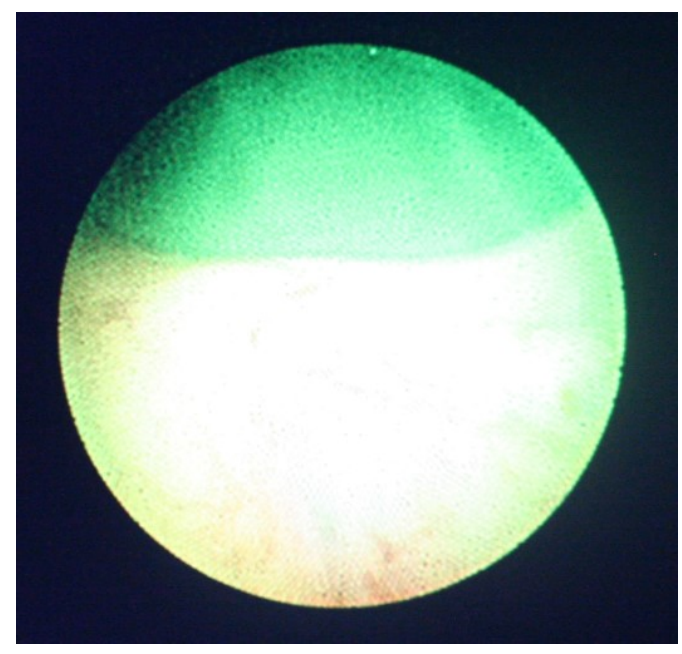

Fig. 1D. The green fluid flow in the abdominal cavity from the urinary bladder after the experimental perforation

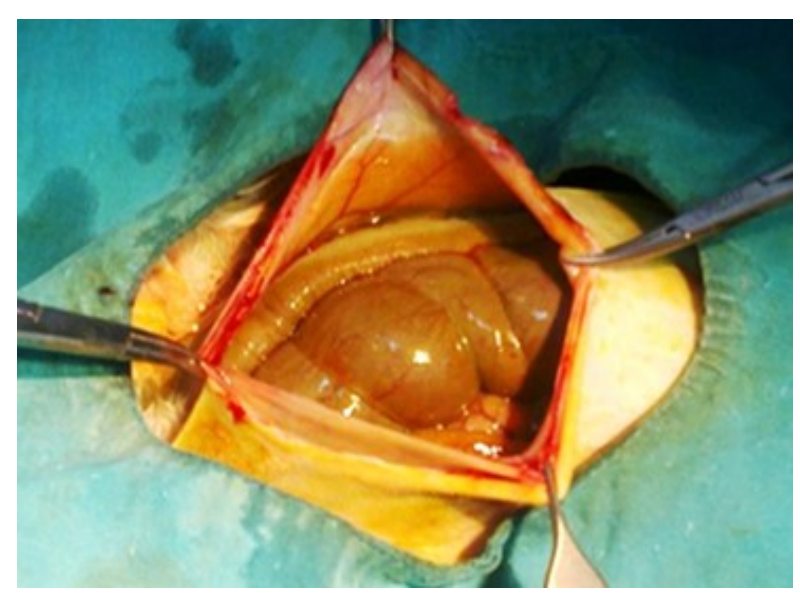

Fig. 2. Laparotomy. All internal organs stained yellow-green

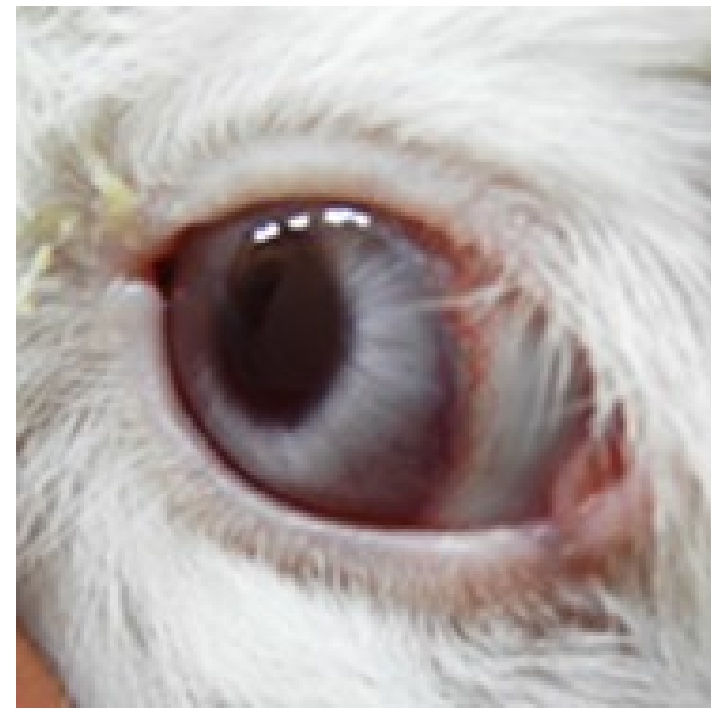

Fig. 3A. No discoloration of the conjunctiva or sclera in retrograde fluorescein group 


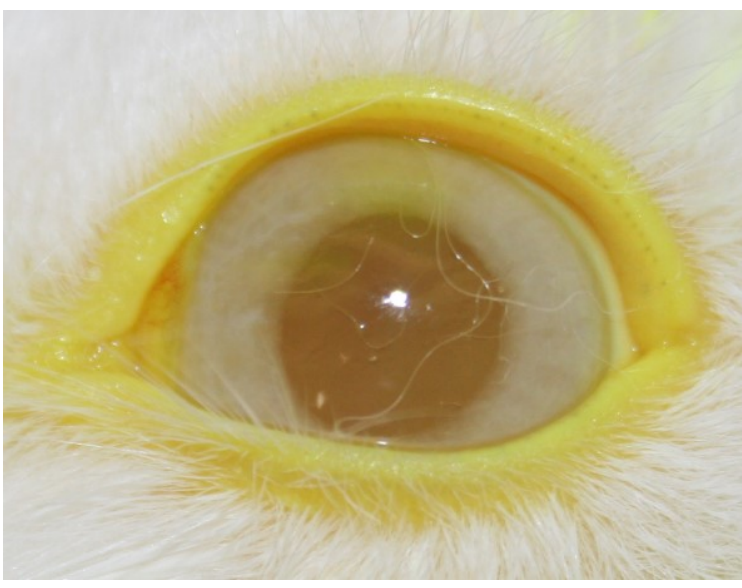

Fig. 3B. Yellow discoloration of conjunctiva and sclera in intravenous fluorescein group

\section{Discussion}

Urolithiasis and urinary bladder rupture which occur due to urolithiasis are common in male ruminants and carnivores $(1,4,8,9,13,15,16,19)$ and in mares (10). Bladder rupture is a condition that requires urgent surgical intervention. It is vital to diagnose rupture within a few hours and treat it expeditiously $(4,9,10$, 15). In this study, a new method was defined. The method is cheap and easy to apply and is an alternative to routine diagnostic methods. Bladder ruptures can be diagnosed with this method in a short time.

Intravenous fluorescein angiography has been used for many years for the purpose of diagnosis of vitroretinal diseases $(2,20)$. However, Zimmern et al. (20) used this technique to diagnose neovascularity, tumours, ischaemic tissues, and hyperaemia of vessels of bladder mucosa in humans. In our study, it has been proved that fluorescein reaches the abdominal cavity and can be used as a marker giving yellow-green colour. Zimmern et al. (20) used $2.5-5.0 \mathrm{~mL}$ of $10 \%$ fluorescein and did not see any side effects such as anaphylaxis, toxication, or permanent colour changes in their patients.

In the present study, the intravenous fluorescein dose of $0.5 \mathrm{~mL}$ was found to be sufficient for diagnosis; however, in order to determine the optimal doses in the other animal species, further studies are needed.

Adverse effects other than discoloration are iatrogenic due to faulty injection and allergic reactions that have rarely been reported (14). The overall risk of death from fluorescein angiography has been estimated to be $1 / 222000(6,11,14,20)$. In our study, yellow discoloration of mucosa, conjunctiva, and sclera was seen after intravenous injection of fluorescein. This effect was consistent with the adverse effects reported in the literature $(6,14,20)$. Advantages of fluorescein are that it is non-toxic, rapidly metabolised, and has minimum adverse effects. It can be said that further studies are necessary to find the IV minimal doses of fluorescein in order to remove some reversible side effects.

Since Cipolla et al. (3) had not demonstrated precisely the peritoneal absorption of fluorescein administered via retrograde catheterisation in rats with experimentally perforated bladders, they centrifuged the blood and determined its fluorescein content. These researchers also reported that sodium fluorescein given via retrograde catheterisation to rats with bladder mucosa injury changed the permeability in the urinary bladder. In the present study, fluorescein administered via the retrograde technique was also found in the abdominal cavity as yellow-green fluid. It is thought that the retrograde fluorescein technique can also be used to diagnose bladder rupture if there is no obstruction in the urethra. However, the introduction of the catheter and the possibility of urethral obstruction were considered disadvantages in terms of timeefficiency.

Positive contrast cystography, computed tomography, and ultrasonography are also used to diagnose urinary bladder rupture $(5,12,18)$. Retrograde positive contrast cystography is rarely rewarding in animals if there is an obstruction in the urethra or urolithiasis. Radiography does not give an exact result when hindered by size of the animal (19). It has been demonstrated that intravenous urography is unacceptable due to high false negative results, with rate of $64 \%-84 \%$ in the diagnosis of bladder rupture in humans (12). Excretory urography is sufficient for diagnosis of renal and ureteral diseases but is not for urinary bladder diseases (18). Thus, this method is not recommended for diagnosis of bladder rupture. Several of the methods mentioned above can be used to diagnose urinary bladder rupture, but administration of fluorescein is extremely simple, provides results in a short time, and is an alternative to other methods very suitable for emergency situations.

The definitive diagnosis of bladder rupture is possible by determining electrolytes in samples of urine that is aspirated from the abdominal cavity after bladder rupture in cattle and horses $(10,19)$. But this method is safe only in a window of a few hours after the rupture (19). In contrast, reliable results are provided at any time if the fluorescein method is used. In our study, animals with full bladders were preferred for administration of fluorescein, but in practice, the normal bladder repletion should be achieved with $0.9 \%$ $\mathrm{NaCl}$ for the paracentesis procedure if the urinary bladder is empty because of the rupture.

In conclusion, we can use fluorescein as a marker in diagnosis of bladder ruptures, such that if there is no bleeding or intestinal content in the abdominal cavity, although a smoky yellow-green image presents, it can be suspected. If there is doubt, laboratory examination of an abdominal fluid sample is essential. The size of the dose should be determined for animals of different species, weights, and sizes in future studies. We believe that the fluorescein method is simple and cheap and 
provides fast results in emergency situations or for veterinarians working under field conditions. It was found that this method is useful and reliable for diagnosis of bladder rupture in animals.

Conflict of Interests Statement: The authors declare that there is no conflict of interests regarding the publication of this article.

Financial Rights Statement: This study was not supported by any research grant or foundation.

Animal Rights Statement: The experimental protocol was approved by the Local Ethical Committee for Animal Experiments of the Kafkas University.

\section{References}

1. Amarpal K.P., Aithal H.P., Pawde A.M., Paratap K., Gugjoo M.B.: A retrospective study on the prevalence of obstructive urolithiasis in domestic animals during a period of ten years. Adv Anim Vet Sci 2013, 1, 88-92.

2. Bearelly S., Rao S., Fekrat S.: Anaphylaxis following intravenous fluorescein angiography in a vitreoretinal clinic: report of 4 cases. Can J Ophthalmol 2009, 44, 444-445.

3. Cipolla A.F., Khedroo L.G., Casella P. A.: Fluorescein test for rupture of urinary bladder. Surgery 1953, 33, 102-106.

4. Connell R., Whiting F., Forman S.A.: Silica urolithiasis in beef cattle I. Observation on its occurrence. Can J Comp Med 1959, 23, 41-46.

5. Cote E., Carroll M.C., Beck K.A, Good L., Gannon K.: Diagnosis of urinary bladder rupture using ultrasound contrast cystography: in vitro model and two case-history reports. Vet Radiol Ultrasound 2002, 43, 281-286.

6. Dithmar S., Holz F.G.: Physical and chemical basics of fluorescence angiography. In: Fluorescence Angiography in Ophthalmology Edited by Öncel M., Avrupa Tıp Kitapçılık, Istanbul, Turkey, 2009, pp. 1-3.

7. Eichel L., Scheidweiler K., Kost J., Shojaie J., Schwarz E., Messing E., Wood R.: Assessment of murine bladder permeability with fluorescein: validation with cyclophosphamide and protamine. Urology 2001, 58, 113-118.
8. Ewoldt J.M., Jones M.L., Miesner M.D.: Surgery of obstructive urolithiasis in ruminants. Vet Clin North Am Food Anim Pract 2008, 24, 455-465.

9. Gugjoo M.B., Zama M.M.S., Amarpal, Mohsina A., Saxena A.C., Sarode I.P.: Obstructive urolithiasis in buffalo calves and goats: incidence and management. J Adv Vet Res 2013, 3, 109-113.

10. Jones P.A., Sertich P.S., Johnston J.K.: Uroperitoneum associated with ruptured urinary bladder in a postpartum mare. Austr Vet J 1996, 74, 354-358.

11. Knowles S.R., Weber E.A., Berbrayer C.S.: Allergic reaction to fluorescein dye: successful one-day desensitization. Can J Ophthalmol 2007, 42, 329-330.

12. Lynch D., Martinez Piñeiro L., Plas E., Sterafetinidis E., Turkeri L., Hohenfellner M.: Guidelines on Urological Trauma, European Association of Urology (2006) http://www.uroturk. org.tr/files/UrologicalTrauma.pdf (accessed 14 February 2013)

13. Parrah J.D., Moulvi B.A., Athar H., Bhat M.I., Handoo N.: Perioperative observations during surgical management of bovine obstructive urolithiasis. J Res Develop 2012, 12, 95-104.

14. Quillen D.A., Blodi B.A.: Ancillary testing for retinal and choroidal diseases. In: Clinical Retina, edited by Quillen D.A., Blodi B.A., Michigan, USA. 2002, p. 16

15. Seddek A.M., Bakr H.A.: New surgical technique for treatment of obstructive penile urethrolithiasis without interference with breeding capability. Clinical study on 25 calves. Pakistan Vet $\mathrm{J}$ 2013, 33, 385-387.

16. Suresh Kumar R.V., Veena P., Sankar P., Dhana-Lakshm N., Kokila S.: Urolithiasis in a buffalo calf - A case report. Buffalo Bull 2011, 30, 222-225.

17. Wallace M.B., Meining A., Canto M.I., Fockens P., Miehlke S., Roesch T., Lightdale C.J., Pohl H., Carr-Locke D., Löhr M., Coron E., Filoche B., Giovannini M., Moreau J., Schmidt C., Kiesslich R.: The safety of intravenous fluorescein for confocal laser endomicroscopy in the gastrointestinal tract. Alim Pharmacol Therap 2010, 31, 548-552.

18. Weeren P.R., Klein W.R., Voorhout G.: Urolithiasis in small ruminants II. Cysto-urethrography as a new aid in diagnosis. Vet Quart 1987, 9, 79-83.

19. Wilson D.G., MacWilliams P.S.: An evaluation of the clinical pathologic findings in experimentally induced urinary bladder rupture in pre-ruminant calves. Can J Vet Res 1998, 62, $140-143$.

20. Zimmern P.E., Laub D., Leach G.E.: Fluorescein angiography of the bladder: Technique and relevance to bladder cancer and interstitial cystitis patients. J Urol 1995, 154, 62-65. 